University of Denver

Digital Commons @ DU

Counseling Psychology Faculty Scholarship

Counseling Psychology

$5-2018$

\title{
Utilization of Telehealth Technology in Addiction Treatment in Colorado
}

\author{
J. Michael Faragher \\ University of Denver, Mike.Faragher@du.edu \\ Ying Shan Doris Zhang \\ University of Alberta \\ Virginia Low \\ University of Denver \\ Demi Folds \\ University of Denver \\ Mita Johnson \\ Walden University
}

Follow this and additional works at: https://digitalcommons.du.edu/counselingpsychology_faculty

Part of the Counseling Psychology Commons, Other Medicine and Health Sciences Commons, Substance Abuse and Addiction Commons, and the Telemedicine Commons

\section{Recommended Citation}

Faragher, J. M., Zhang, Y. S. D., Low, V., Folds, D., \& Johnson, M. (2018). Utilization of telehealth technology in addiction treatment in Colorado. Journal of Technology in Behavioral Science. DOI: 10.1007/ s41347-018-0057-3

This work is licensed under a Creative Commons Attribution 4.0 License.

This Article is brought to you for free and open access by the Counseling Psychology at Digital Commons @ DU. It has been accepted for inclusion in Counseling Psychology Faculty Scholarship by an authorized administrator of Digital Commons@DU. For more information, please contact jennifer.cox@du.edu,dig-commons@du.edu. 


\section{Utilization of Telehealth Technology in Addiction Treatment in Colorado}

\section{Comments}

The final version of the article is freely available at https://rdcu.be/M9nA

This is a post-peer-review, pre-copyedit version of an article published in Journal of Technology in Behavioral Science. The final authenticated version is available online at: https://doi.org/10.1007/ s41347-018-0057-3

\section{Publication Statement}

Copyright held by the author or publisher. User is responsible for all copyright compliance 
Utilization of Telehealth Technology in Addiction Treatment in Colorado

\author{
J. Michael Faragher \\ Counseling Psychology Dept. | University of Denver \\ Rm. 225 | 1999 East Evans Avenue | Denver, CO 80208-1700 \\ 303-434-6981 | mike.faragher@du.edu | ORCID: 0000-0002-2252-3713 \\ Ying Shan Doris Zhang \\ Department of Psychology | University of Alberta \\ P-217 | Biological Sciences Building | Edmonton, Alberta, Canada T6G 2E9
}

\author{
Virginia Low \\ Counseling Psychology Dept. | University of Denver \\ 1999 East Evans Avenue | Denver, CO 80208-1700 \\ Demi Folds \\ Counseling Psychology Dept. | University of Denver \\ 1999 East Evans Avenue | Denver, CO 80208-1700 \\ Mita Johnson \\ Walden University | PO Box 4297, Evergreen, CO 80437 \\ Acknowledgments \\ Riley Cochran, Alli Tellez
}

Ethics Approval: This research has been approved by the university's Institutional Review Board (IRB), Human Subjects Protection Program, Office of Research Compliance, and has followed procedures in accordance with the ethical standards laid down in the 1964 Declaration of Helsinki and its later amendments.

Informed consent: The voluntary nature of this study was stated clearly in the text and all persons gave their informed consent prior to their inclusion in the study.

Conflict of interest: None of the authors have conflicts of interest and authors have full control of all primary data and agree to allow the journal to review data if requested. 


\begin{abstract}
The growing presence of electronic technology in the health service professions is redefining the boundaries of counseling services. Commonly referred to as telehealth, utilization of electronic communication strategies to expand connectedness has opened new frontiers in behavioral health through applications ranging from digital phones, interactive video sessions, to virtual supervision. Substantial research suggests that telehealth is generally equal in effectiveness to traditional forms of treatment, especially for those individuals struggling with substance abuse problems. Unfortunately, research also suggests that telehealth is often underutilized when it comes to providing addiction treatment services. Telehealth trends in Colorado were examined using a Telehealth Survey consisting of 29 items. Participants consisted of 125 members of the Colorado Association of Addiction Professionals. Similar to research published elsewhere, $65 \%$ reported that they do not currently use telehealth technologies. Furthermore, findings illustrated that actual use can vary by ethnicity, age group, type of organization, as well as service location. Participants' reluctance to implement telehealth is related to concerns associated with training, confidentiality, clinician/staff acceptance, and reimbursement. Future research, employing both quantitative and qualitative methods, is recommended to further explore both the challenges and solutions to promote telehealth use, as well as methods to expand relevancy and awareness. Keywords: telemedicine, telehealth, addiction treatment, substance misuse, e-therapy, treatment technology
\end{abstract}


Introduction

No innovation has revolutionized the delivery of behavioral health services more than electronic information and telecommunication technology. From texting on smartphones to realtime virtual therapy sessions over the internet, advances in technology have greatly diminished the requirement of face-to-face interactions in the provision of behavioral health counseling services. The depth and breadth of these technologies are rapidly expanding across all modalities. The federal Health Resources and Services Administration (HRSA) defines telehealth as:

"The use of electronic information and telecommunications technologies to support longdistance clinical health care, patient and professional health-related education, public health and health administration. Technologies include video conferencing, the internet, store-and-forward imaging, streaming media, and terrestrial and wireless communications.” (https://www.hrsa.gov/ruralhealth/telehealth/index.html) Telehealth services that include in-person video conferencing are called realtime/immediate services, or synchronous services, while services provided through e-mail or online surveys are called store-and-forward services, or asynchronous services (Baca, Alverson, Knapp-Manuel, \& Blackwell, 2007; NASADAD, 2009). Synchronous services allow for realtime interaction between the client and the healthcare professional and include services such as, video conferencing, webcam assisted, and telephone communication (NASADAD, 2009). Alternatively, asynchronous services are typically used to reciprocally forward or transfer health related data between the healthcare professional and the client. These services include exchanging emails (digital images or other medical concerns) and conducting online surveys (NASADAD, 2009). 
The growing presence of these telehealth services in health care professions has become ubiquitous in the professional life of healthcare providers. Even a decade ago, it was recognized that services using technologies, such as telephone, teleconferencing, and internet services were rapidly evolving into important elements of counseling services (American Psychological Association, 1997). More recently, telehealth services are increasingly integrated into rural/frontier areas as well as urban services configured to work with underserved populations to provide more effective and accessible care for those with limited access to health care services (Batastini, King, Morgan, \& McDaniel, 2016). Medicare data on telehealth utilization collected from 2004 to 2014 showed a $45.1 \%$ national annual growth rate, an increase from 2,365 to 87,120 , of telehealth visits for rural patients with a mental health diagnosis (Mehrotra, et al., 2017). Additionally, the data showed a $49.3 \%$ annual growth rate, an increase from 1,040 to 50,050, visits for rural patients with serious mental illness (Mehrotra, et al., 2017). The evolving importance of telehealth was highlighted by HRSA in 2012 through the creation of the National Telehealth Policy Resource Center (NTRC-P) with twelve federally funded telehealth regional resource centers. These centers provide support for the development of advanced telehealth technologies.

A growing body of research shows that telehealth is as effective as traditional forms of treatment. For example, research conducted at a methadone maintenance treatment facility integrated counseling services with web-based psychosocial and behavioral intervention modules (Marsch, 2013). The results indicated that this inclusion of telehealth services was as effective as other counseling services. The provision of telehealth services also resulted in "significantly greater rates of objectively measured opioid abstinence" (Marsch, 2013, p. 49). Moreover, Batastini and colleagues conducted a meta-analysis on telehealth services within the criminal 
justice system among substance use disorder clients (2016). These findings also showed that telehealth outcomes were "at least comparable with in-person outcomes" (Batastini et al., 2016, p. 27). This meta-analysis consisted of five studies which included clients with substance abuse and clients within the criminal justice system, all of which had a comparison group for reference. Findings from this meta-analysis were consistent with comparable research which supported observations that telehealth services are comparable to traditional counseling services (Batastini et al., 2016).

As well, an additional meta-analysis on the effectiveness of telehealth services in substance abuse treatment produced results similar to the above studies. This research included a thorough review of 145 separate studies assessing the effectiveness of telehealth services that targeted smoking cessation, alcohol abuse, illicit drug abuse, and gambling (Ohinmaa, Chatterley, Nguyen, \& Jacobs, 2010). This meta-analysis noted that offering more individualized programs can improve an individual's outcomes in substance abuse treatment as well as the suggestion that including internet applications to existing treatment can be beneficial (Ohinmaa et al., 2010). The majority of the studies included in Ohinmaa and colleagues' meta-analysis were large-scale randomized controlled trials. The strength of this research approach, along with the previous mentioned studies, provides compelling evidence of the importance of increasing the presence of telehealth interventions.

Telehealth interventions have also been used to treat gambling addictions while simultaneously reducing co-occurring psychological symptoms. For instance, a Cognitive Behavioral Therapy (CBT) web based intervention was shown to significantly reduce problem gambling and symptoms of comorbid disorders (Carlbring \& Smit, 2008). Programs combining telephone and internet based treatment have also demonstrated significant effects in reducing 
pathological gambling symptoms, as well as cognitive distortions and psychological distress (Myrseth, Brunborg, Eidem, \& Pallesen, 2013). Although research suggests only 10\% of problem gamblers seek treatment, having access to telehealth services, including internet based interventions, may lower barriers to obtaining treatment for people struggling with addiction (Myrseth et al., 2013).

The benefits of telehealth services are supported by an ever-growing body of evidence. Ways in which they are implemented continue to grow in parallel with the evolution of technological capabilities and software development. It is increasingly evident that services delivered via technology eliminate many barriers to receiving health care services, such as physical distance, disabilities, and social stigmas (Curie, 2005), as well as the transportation challenges faced by many (Batastini et al., 2016). Historically, the above barriers have prevented many people from accessing health care services, compounded by the added financial burdens associated with travel time and work absence. Preliminary studies have also illustrated that health care provided through telehealth can be an effective mode of treatment by overcoming barriers associated with cost and access (Curie, 2005).

Telehealth services have demonstrated benefits within rural/frontier and urban services due to the greater availability of services within remote regions and easier accessibility of information (Gagnon, Duplantie, Fortin, \& Landry, 2006). Some particular challenges the individuals within these underserved areas face might include: distant location of their homes relative to treatment facilities; potential complications if one has a physical disability; limited access to their own transportation or public transportation; as well as the rising costs of healthcare services (Batastini et al., 2016; Smalley, Yancey, Warren, Naufel, Ryan, \& Pugh, 2010). In health care systems, particularly medical health practices, telehealth technologies 
demonstrated effectiveness in increasing the participation of patients without requiring patients to travel long distances to their health practitioner's treatment site. Even back in the year of 2012, it was reported that "[e]very year in the United States, about 10 million patients receive telemedicine services" (Linkous, 2012, p. 17). In California, telehealth is seen as an advancement in medical care and a has provided a reduction in medical care costs, which was an identified concern in this study (Leach, 2009).

Additionally, frontier and rural communities frequently encounter unique challenges regarding access to mental health and substance abuse treatment services (Benavides-Vaello, Strode, \& Sheeran, 2013). Telehealth can help break through the roadblocks of limited access often found in frontier and rural communities through reduced travel time, easier access for those individuals with physical disabilities, decreased family disruption as a result of shortened travel time or lessened financial concerns, as well as fewer missed appointments (Benavidas-Vaello et al., 2013). Furthermore, telehealth can also reduce long waitlists, lessen prohibitive costs, and can protect against the perceived shame and stigma that may deter individuals from seeking help (Evans \& Delfabbro, 2005).

Regrettably, current research points to mental health counselors in general falling behind other behavioral health professions in the implementation of telehealth services. Data collected in 2014 showed that of the national Medicare providers using telehealth to deliver services to rural patients with a serious mental illness, $65.3 \%$ were psychiatrists, $19.9 \%$ were nurse practitioners, and only $8.8 \%$ were clinical psychologists (Mehrotra, et al., 2017). Even further, the specific area of addiction counseling treatment services falls short as compared to other behavioral health services in the use of technology in healthcare delivery (Ohinmaa et al., 2010; Enos, 2015). The addiction treatment profession is struggling to close the gap between these 
other areas of medical healthcare and to regain its place in the provision of state-of-the-art treatment services in the healthcare arena. Furthermore, additional research has suggested that, due to privacy laws associated with substance use disorder, there have been limitations leading to underestimation of the number of patients receiving telehealth services due to redacted substance use disorder codes (Mehrotra, et al., 2017). The current study instead collects information from providers rather than searching through Medicare claims or electronic medical record systems.

Considering the well-documented advantages of offering telehealth options as a part of an addiction treatment package, and to close the gap between addiction treatment and other healthcare services regarding the use of telehealth technologies, telehealth trends in Colorado were examined in this study. As a preliminary step, it was determined that a baseline assessment of current telehealth utilization in Colorado must be conducted. To this end, addiction counselors were surveyed regarding the status of the current utilization of telehealth technologies in Colorado.

The objectives of this current study intend that readers will 1) learn about the current trends in the utilization of telehealth including types of services, methods, and settings, 2) understand service providers' knowledge and rationale for using telehealth services, 3) discover limitations and concerns providers have about using telehealth services, 4) become aware of the challenges faced in the implementation of novel technological interventions, 5) become aware of areas of competence needed to use/improve telehealth services, and 6) be provided a foundation, from this pioneer study, for expanded research in other states and countries.

\section{Method}

\section{Participants}


Participants were 125 treatment professionals offering addiction healthcare services in the state of Colorado. Among the 125 participants, 4 participants could possibly have been employed outside of the state. In this sample, 82 individuals identified as female and 43 identified as male. Two participants indicated their age as less than 30 years, 21 participants indicated their age as between 31-41 years, 26 indicated their age as between 42-50 years old, 38 indicated as between 51-60 years, and 38 indicated as more than 60 years old.

In terms of ethnicity, 95 participants identified as White, 14 identified as Hispanic/Latino(a), 6 identified as American Indian/Alaska Native, 5 identified as African American, 4 identified as "other", and 1 participant preferred not to identify.

Fifty-four participants indicated working in addiction treatment programs (inpatient, outpatient, and residential), 40 reported employment in private practices, 17 indicated reported employment in other types of organizations (e.g. advocacy organizations, faith-based organizations, and recovery support programs, etc.), 13 reported employment in correctional facilities, 10 reported employment in community mental health centers, 6 reported employment in hospitals/hospital-affiliated clinics, 5 reported employment in community health centers, 3 reported employment in schools/university-based clinics, and 2 reported employment in tribal health clinics/centers.

Regarding job titles and primary positions, 66 participants identified as clinicians, 31 identified as others, such as behavioral health workers, correctional supervisors, and case managers, etc., 13 identified themselves as administrators, 13 identified as clinical supervisors, and 2 participants identified themselves as researchers.

In terms of services offered, 102 participants provided substance abuse treatment services, 79 provided mental health treatment services, 47 provided recovery support services, 
and 28 provided other types of services such as marriage and family therapy, trauma informed treatment, as well as grief and loss, etc. Regarding service areas in the state of Colorado, about 94 participants stated that their services are provided in urban areas, and 29 stated that their services are provided in rural areas.

Regarding years of experience working in substance abuse treatment/recovery, 6 participants selected less than a year, 21 chose between 1-5 years, 32 chose 5-10 years, 20 chose 10-15 years, 12 chose $15-20$ years, and 34 indicated more than 20 years of experience.

\section{Measure}

The Telehealth Survey. The 29-item Telehealth Survey was reviewed by the executive staff of National Frontier and Rural Addiction Technology Transfer Center (NFAR ATTC), Colorado Association of Addiction Professionals (CAAP), Association for Addiction Professionals (NAADAC), and Substance Abuse and Mental Health Services Administration (SAMHSA) Region 8. Suggestions offered from these reviewers were incorporated into the survey during the development process before arriving at the final format.

The Telehealth Survey is divided into three sections. The first section was designed to collect respondents' background information, including their demographic data. Sample items include "What is your job title or primary position," "Please identify your gender," and "Please select the ethnic group you identify the most with." The last question in this section asks respondents whether they are currently using telehealth technologies (e.g. computer, smartphone, tablet, other mobile device) to deliver services to their clients. Upon selecting the response "Yes," respondents were allowed entry to the final two sections of the survey. On the other hand, by selecting the response "No," respondents would be directed to the end of the survey, which expressed appreciation of their participation. 
The second section is designed to gather information on the current usage of telehealth services, such as specific telehealth services utilized. Sample survey items in this section include "How many years of experience do you have working with telehealth," "How are you reimbursed for providing services utilizing telehealth technologies," and "Which of the following activities do you currently utilize with telehealth technologies."

The last section of the survey gathers additional telehealth services' information, such as training in technology, supervision protocols, confidentiality protocols, attitudes towards using telehealth services, as well as future directions and limitations for telehealth use. Sample items include "What topics were you trained on for delivering services using telehealth technologies," "What type of encryption are you using to protect client data," "What is your biggest concern with telehealth services."

Participants responded to the survey items by a mixture of choosing single and/or multiple response(s), providing open-text responses, as well as providing ratings on two 5-point Likert Scales, from 1 (Not comfortable at all/Strongly disinterested) to 5 (Completely comfortable/Strongly interested). Please refer to Appendix A for details regarding this survey.

\section{Procedure}

The entire survey was analyzed using Qualtrics, an online research software that was established in 2002 in Utah, United States (“Qualtrics Research Core,” 2017). An anonymous link was then generated for interested participants to participate in the Telehealth Survey. In order to capture the most comprehensive view of addiction counselors in the state of Colorado, the survey link was distributed twice through the Colorado Association of Addiction Professionals (CAAP)'s membership database containing 387 active members, as well as posted four times on LinkedIn (from which 5,100 followers of CAAP were allowed access). Those 
receiving an email invitation were notified their participation was completely voluntary. Upon opening the link in the email invitation, participants were directed to the introduction page of the survey, which reads, "You might have noticed the expanding role that technology is playing in behavioral health. In order to construct a statewide plan moving forward, it is important to define the current status of telehealth utilization in Colorado. To this end, we are attempting to identify the current technology usage patterns in the field of addictions treatment in Colorado. We greatly appreciate your input and support by completing this short survey. Study results will be provided to you upon completion of this research project." Interested participants were then able to proceed through the survey by selecting the best response(s) to each survey item. Those subjects accessing the survey through the LinkedIn announcement have responded to an invitation to voluntarily complete the survey, and were also directed to the opening paragraph regarding participation as described above. Upon completion, the surveys were submitted directly to the research team via Qualtrics.

\section{Results}

Reports illustrating patterns and trends of telehealth usage were generated by Qualtrics and analyzed from descriptive data. SPSS was considered as an analysis tool as well, but was deemed inappropriate for the descriptive data contained in this study.

Regarding telehealth use, survey results showed that 81 participants reported they are not currently using telehealth services, while 44 participants indicate they are currently using telehealth for clinical services. The results gathered from the participants revealed a reluctance to use telehealth services in the addiction profession, with $65 \%$ of participants indicating they do not currently use any form of telehealth service. 
In terms of the association between identified age and telehealth use, results revealed that participants between the ages of 31-41 years showed the lowest telehealth usage compared to other age groups, with $81 \%$ reporting they do not currently use telehealth technologies in their practice. Of the 26 respondents between ages $42-50,68 \%$ reported they do not currently use telehealth services and of the 76 participants who indicated ages greater than $51,61 \%$ reported they do not currently use telehealth to deliver services. Additionally, results revealed of the 2 participants under the age of 31,1 indicated they are not currently using telehealth to deliver services.

Additionally, when the association between race/ethnicity and telehealth use was examined, results showed that $80 \%$ of the 5 African American respondents indicated current usage of telehealth technologies. Of the 95 respondents who identified as White, $68 \%$ respondents indicated current usage of telehealth technologies. Among the 14 Hispanic/Latino(a) respondents, $50 \%$ indicated they are currently using telehealth technologies. Respondents who identified as "Other" showed that $25 \%$ of them are utilizing telehealth technology services and of the 6 American Indian/Alaskan Native respondents, 17\% indicated they currently use telehealth technologies.

Furthermore, upon examining the association between the types of organizations in which respondents were employed and telehealth usage, findings showed that 1 of 2 respondents working in Tribal health clinics currently use telehealth technologies, $46 \%$ of 13 respondents working in correctional facilities indicated current usage of telehealth technologies, $38 \%$ of 17 respondents working in other types of organizations indicated current use of telehealth technologies, $35 \%$ of 54 respondents working in addiction specific programs currently use telehealth technologies, $33 \%$ of 40 respondents in private practice currently use telehealth 
technologies, $20 \%$ of 15 respondents working in community health/mental health settings currently use telehealth technologies, $17 \%$ of 6 respondents working in hospital settings currently use telehealth technologies, and $0 \%$ of 3 respondents working in school/university settings indicated current use of telehealth technologies.

Participants were asked where services were provided with the option of choosing either "urban" or "rural." Results revealed that out of the 123 participants who responded to the item, $76 \%$ indicated they provide services in urban areas and $26 \%$ provide services in rural areas. Of the 43 participants who reported they are currently using telehealth services, $74 \%$ indicated they are located in urban areas and $26 \%$ are in rural areas. Out of these 43 participants, 39 went on to complete the survey in its entirety.

Results also revealed that among the 39 participants who indicated utilizing telehealth services, $79 \%$ indicated using phone services, $77 \%$ indicated using email, $54 \%$ indicated using text messaging, 38\% indicated using video conference services, $38 \%$ indicated using web-based interventions, $18 \%$ indicated using mobile apps, $8 \%$ indicated using instant messaging, and 5\% indicated using other types of services which were not listed as a choice option in the survey. See Table 1.

Furthermore, as shown in Table 2, when participants were queried about the type of telehealth methods used most frequently to provide services, $77 \%$ indicated using email, $74 \%$ indicated using phone, $38 \%$ using text messaging, 23\% using web-based interventions, $20 \%$ indicated they are using video conferencing, $15 \%$ indicated using mobile apps, $13 \%$ indicated using instant messaging, and 3\% using other services not listed.

Additionally, participants were asked which form of telehealth services, Synchronous (real time, video conferencing) or Asynchronous (store and forward), were used most frequently 
to provide addiction treatment services. Results showed that of 39 respondents, $72 \%$ indicated they are using Asynchronous forms of telehealth and $28 \%$ indicated they are using Synchronous forms of telehealth services.

The reasons providers are using telehealth services were gathered by querying the purposes of utilizing telehealth. As displayed in Table 3, 49\% of participants are using telehealth for assessment, 44\% for counseling, 44\% for client education, $36 \%$ for clinician/ staff training, $33 \%$ for recovery support, $31 \%$ for case management, $23 \%$ for screening purposes, $21 \%$ for continuing care, $18 \%$ for clinical supervision, $13 \%$ for medical management, and $5 \%$ indicated they are using telehealth for other activities not listed.

Participants who reported current use of telehealth services were asked about reimbursement for using telehealth services. Results illustrated in Table 4 show $46 \%$ of participants indicated they do not receive any reimbursement for telehealth technologies, $33 \%$ receive reimbursement from private pay, $15 \%$ from Medicaid, 15\% from sliding scale, 13\% from other reimbursement methods not listed, 10\% from private insurance, and 5\% from Medicare. Among the participants who do receive reimbursement, the largest amount is received from private pay and private insurance, and a lesser amount is received from insurance providers.

Participants who indicated current use of telehealth services were also asked to identify the topics in which they received training on for delivering services using telehealth technologies. Results highlighted in Table 5 reveal that $83 \%$ of participants identified being trained in confidentiality issues, $69 \%$ trained in obtaining consent, $69 \%$ trained in ethics and safe practices, $66 \%$ trained in state-specific regulations/ requirements, $55 \%$ were trained in how to use the equipment/ technology, 55\% trained in licensing and credentialing, 55\% trained in client 
management, and $31 \%$ trained in reimbursement documentation, storage, and retrieval of records.

When investigating barriers to using telehealth, participants were asked to select their foremost concern(s) with telehealth services. As seen in Table 6, results show $41 \%$ were concerned with clinician/staff acceptance, $41 \%$ concerned with confidentiality, $21 \%$ concerned about training, $21 \%$ concerned with client acceptance, $21 \%$ concerned with using the technology and equipment, $17 \%$ concerned with billing/reimbursement, $14 \%$ participants were concerned about the cost of services, $14 \%$ concerned with credentialing, and $14 \%$ concerned with other options not listed.

Additionally, participants were asked to rate the significance of each concern. Health Insurance Privacy and Portability Act (HIPPA) and clinician/staff acceptance were rated as the most significant barriers to telehealth utilization. Detailed results are displayed in Table 7.

Lastly, participants were invited to share their thoughts on "What other information would be helpful to enhance your understanding of telehealth?” Participants responded with answers to this open-ended question such as "free webinars," "provide more resources," "client referral," "encryption software," "understanding encryption," "telehealth training," "cost," "training on all available resources," "ensuring client security," "since regulations have not caught up to technology, additional training on jurisdiction and scope of services would be useful," and "Colorado laws." Participants were also invited to share their thoughts on "What other information/assistance might you need to increase the likelihood for you to use telehealth in the future?" Participants responded with answers to this open-ended question such as "clarification of confidentiality rules with use," "client referral," "education/trainings," "financial assistance," "free trainings," "information on therapeutic issues when using telehealth," "private 
insurance willing to pay," "training," and "various processes telehealth uses in major areas." Results revealed that overall, responses to both questions fall into the domains of "training," "encryption," and "financial."

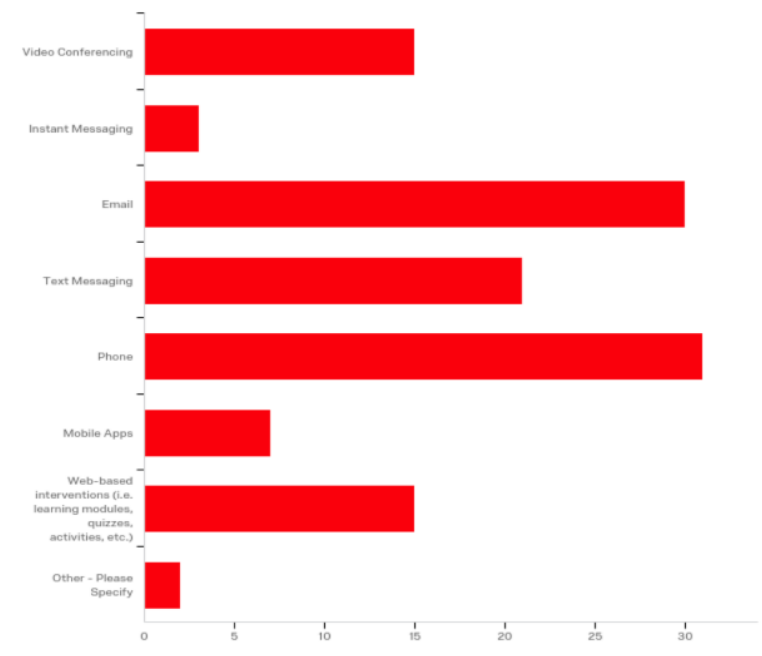

Table 1. Format and frequency of telehealth technologies used

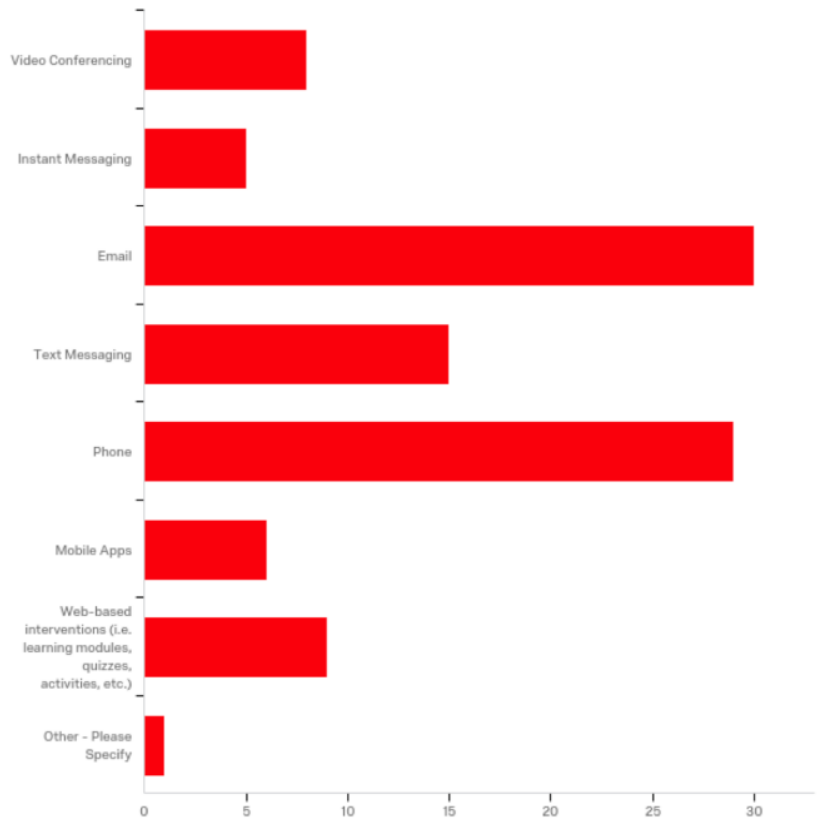

Table 2. Frequency of methods for telehealth used 


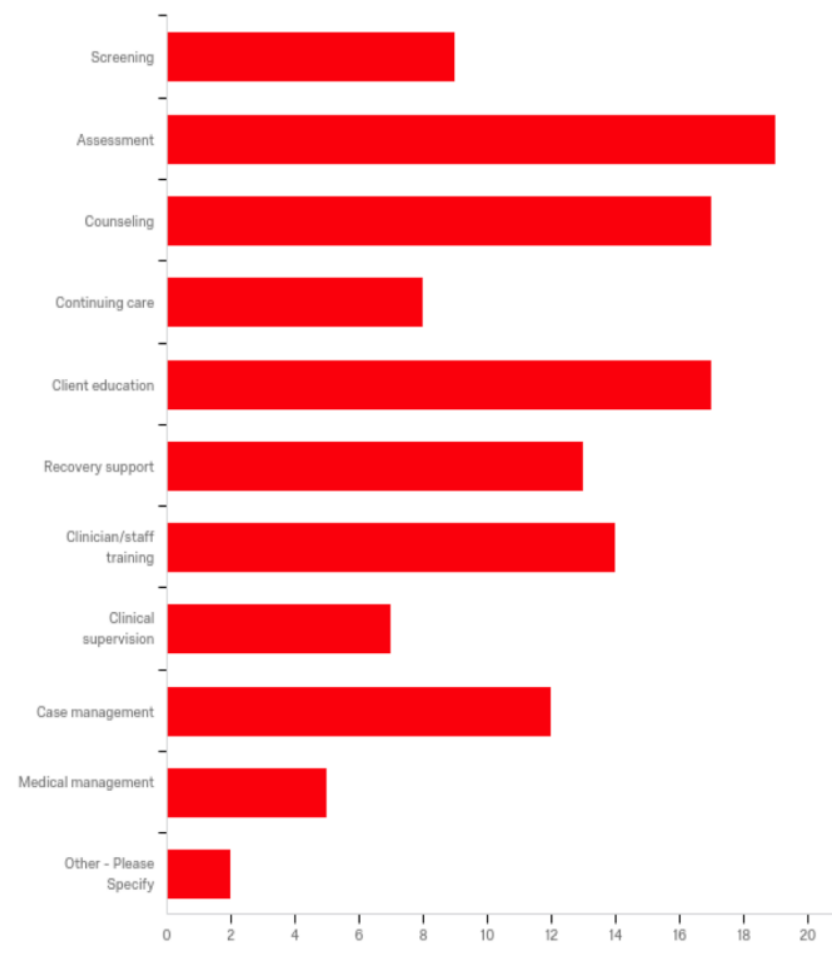

Table 3. Frequency of practices associated with telehealth technologies

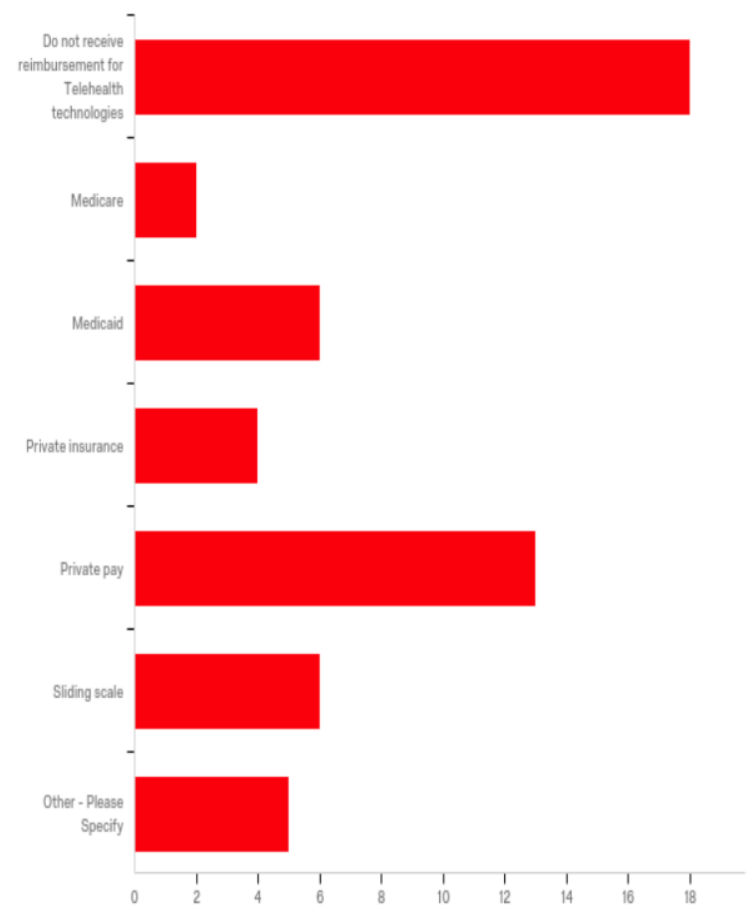

Table 4. Frequency and sources of reimbursement for telehealth services 


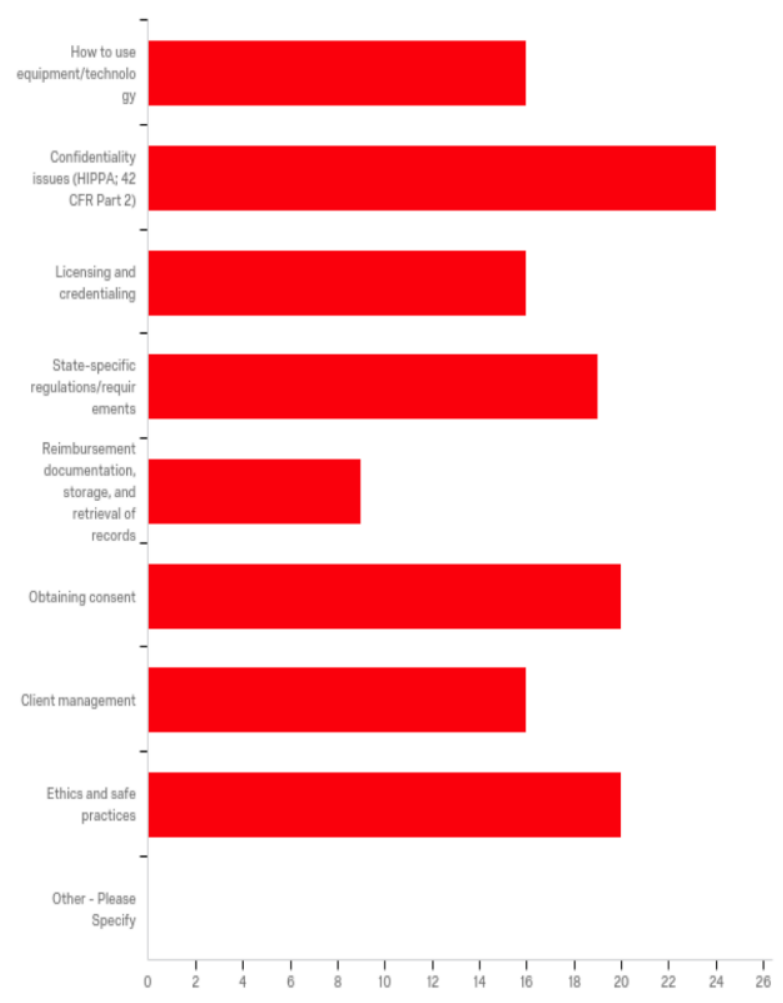

Table 5. Frequency of clinical trainings in telehealth services

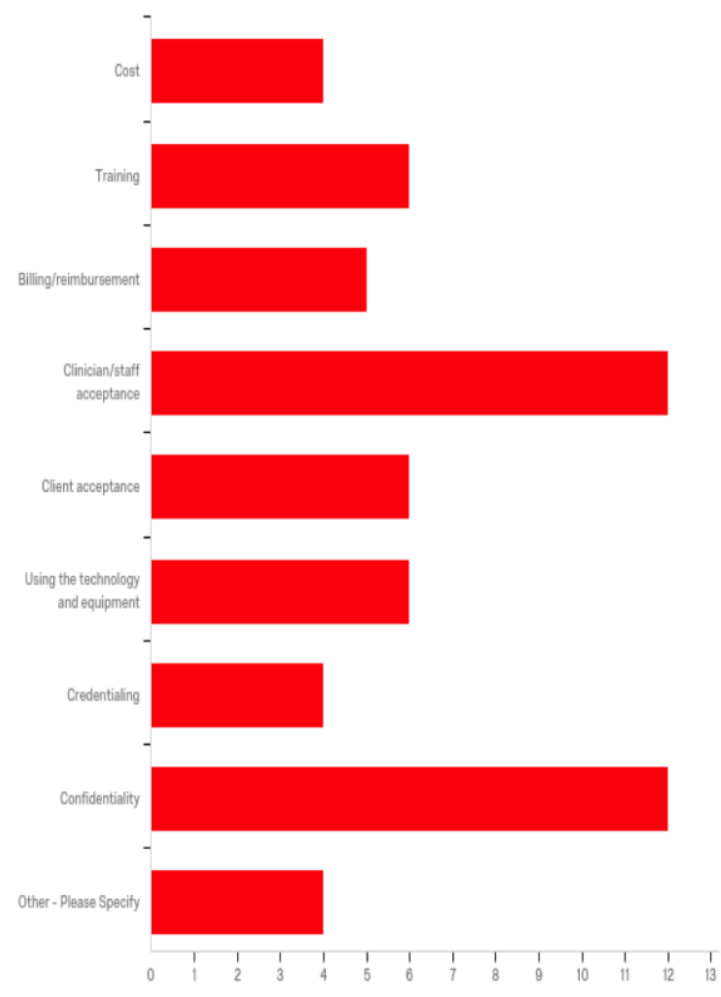

Table 6. Frequency of concerns with utilizing telehealth services 


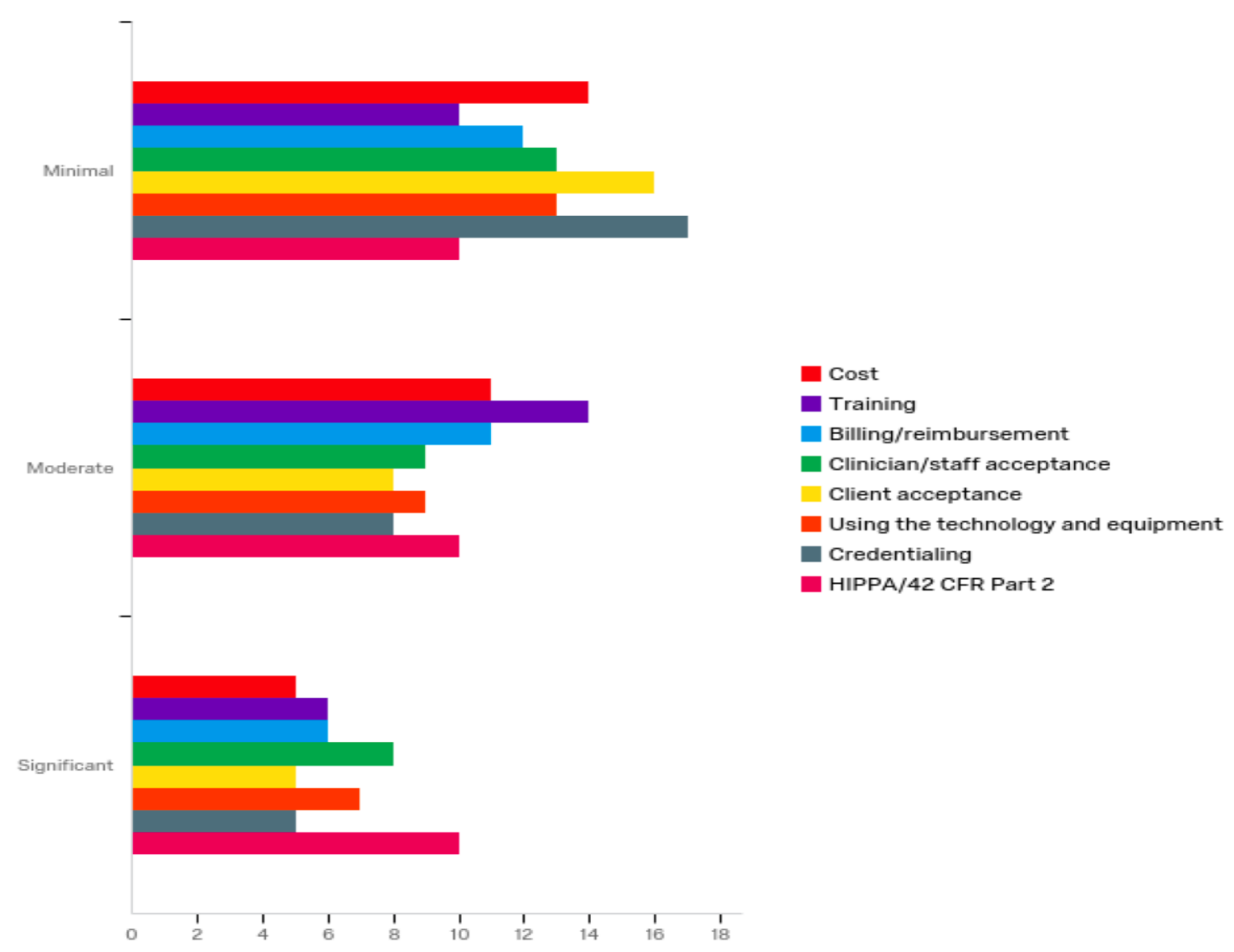

Table 7. Perceived extent to which each domain serves as a barrier to using telehealth technologies to deliver services

\section{Discussion}

Results revealed more than half of the sample reported they did not use telehealth in their provision of addiction treatment services. These findings are consistent with the literature review which suggests that telehealth services in the addiction profession are lagging behind other areas in health care. Results also identified clinic locations in which telehealth services are more or less likely to be implemented. When comparing participants who work in urban or rural areas and are currently using or not using telehealth services, collected data suggested more addiction treatment clinicians reported employment in urban areas than rural areas. However, for each 
location, more than half of clinicians in the study sample reported not using telehealth services. Furthermore, findings illustrated that there were no distinct differences in which type of organization showed lower usage of telehealth relative to the sample size. This may suggest future research could be done to determine barriers within each organization that limit the utilization of telehealth.

Results also revealed that providers are using more Asynchronous forms of telehealth technologies. Though reasons for this preference are unknown, a possible explanation, as suggested by Canale et al. (2016), is that web based interventions (a form of Asynchronous telehealth) are preferred due to concerns around confidentiality and the non-judgmental quality of the intervention that can increase an individual's willingness to share information. Similarly, these interventions can be brief and disseminated to larger groups. Also, these interventions require minimal training and are relatively inexpensive (Canale et al., 2016).

Regarding challenges faced by counselors, results of the current study indicate that barriers to utilization of telehealth services in the field of substance use disorder treatment are mainly associated with concerns around confidentiality, training, financial reimbursement, and clinician acceptance in the workplace. To begin with, data collected around the domain of reimbursement may help to explain why providers are reluctant to utilize telehealth. Results showed $46 \%$ of participants indicated they do not receive any reimbursement for telehealth technologies. Of those that reported reimbursement, most counselors reported receiving reimbursement from private pay. This may suggest that many insurance providers have not fully integrated telehealth reimbursement into their payment system for substance use disorder treatment. It may also indicate a general confusion or lack of knowledge regarding evolving reimbursement protocols. In a broader sense, this may help us understand why telehealth is not 
being implemented more frequently. For example, shared Medicare laws are composed of multifaceted regulations which allow patients to receive telehealth services and providers to receive reimbursement. Currently, Medicare and Medicaid cover clients receiving video conference services in rural areas or while in an originating site as defined by the Centers for Medicare \& Medicaid Services (CMS). Examples of such originating sites include, but are not limited to, hospitals, Community Mental Health Centers, Rural Health Clinics as well as offices of physicians or practitioners. Additionally, in most states, the provider must be licensed in the same state as in which the client resides to receive reimbursement (Mehrotra, et al., 2017).

Moreover, the challenges to reimbursement may also be associated with training deficits. Findings in the current study illustrated that reimbursement/documentation, storage, and retrieval of records ranked the lowest area for training. An interesting finding from this analysis revealed that of 29 respondents, $68 \%$ were not properly trained in reimbursement documentation. This lack of training may also contribute to why providers report not being reimbursed for services. Provider training should include discussion of differing insurance company standards as well as varying state regulations. Providers unaware or not trained in this area may be unable to receive reimbursement from Medicare or other insurance providers.

Furthermore, although results indicate that financial reimbursement and training related to reimbursement are identified preliminary concerns with the implementation of telehealth services, confidentiality and clinician acceptance appear to be the most significant perceived barriers. Within the broad domain including all behavioral/medical health disorders, treatment for substance use disorders faces a unique challenge in the area of confidentiality. Federal statute, 42 CFR Part 2 Final Rule (Knopf, 2016), imposes more severe restrictions on confidentiality of communications and records for substance use disorder patients than do the 
HIPAA requirements associated with all other behavioral/medical disorders. The protections afforded by 42 CFR Part 2 were intended to provide additional protection for substance use disorder clients from possible discrimination in many areas that include housing and employment. The concern was that individuals with substance use disorders would avoid treatment if they thought this information could be used against them. Thus, while HIPAA permits patient disclosure for the purposes of payment for services, 42 CFR Part 2 specifically prohibits this activity without patient consent. Additionally, while HIPAA does not address redisclosure of patient information to third party providers, 42 CFR Part 2 prohibits providers that have received patient records from disclosing these records to a third provider. These more restrictive requirements are considered an additional barrier to sharing patient information by many in the addiction field (McCarty, Rieckmann, Baker, McConnell, 2017). Since many have suggested that the additional layers of required privacy associated with 42 CFR Part 2 for substance use clients impede the delivery of integrated behavioral health solutions (Schaper, Padwa, Urada, Shoptaw, 2016), a somewhat more flexible revision of 42 CFR Part 2, referred to as the Final Rule, took effect on March 21, 2017. Even with this revision, however, SAMHSA has upheld the primary protections of 42 CFR Part 2 possibly resulting in substance use counselors exercising elevated levels of caution in providing information regarding client services.

Additionally, because concerns with HIPAA and confidentiality appear to be prominent among addiction counselors, it raises the question of why more medically focused healthcare treatments have had greater success implementing telehealth services. This might indicate that a lack of knowledge around telehealth services and their specific interventions in the field of substance disorder treatment is contributing to clinical avoidance, as evidenced by higher rates of 
concern around clinician acceptance. This, of course, would surely include an elevated level of guardedness regarding the use of electronic telehealth communications. Perhaps a review of strategies that have successfully addressed these initial concerns among more medically oriented treatments would inform the addiction treatment field. Regardless, it is apparent that a need for trainings and information specific to telehealth services in the field of substance use disorder treatment is necessary to help replace counselor concerns with informed confidence and clinical acceptance.

\section{Conclusion}

Consistent with current investigations conducted nationally, findings from this study indicate that telehealth technologies have not been widely utilized in addiction treatment services in Colorado (Ohinmaa et al., 2010; Batastini et al., 2016). Also, actual use of telehealth can vary by ethnicity, age group, type of organization, as well as service location. Purpose for using telehealth technologies, as well as the specific type of technology used, also differed in relationship to demographic variables. As well, variations were found among telehealth training and reimbursement for clinicians. Moreover, the most frequently reported challenges to utilizing telehealth services include concerns with HIPAA and confidentiality, as well as clinician/staff acceptance. Several of the other commonly reported concerns in using telehealth technologies in clinical services identified issues related to "training," "encryption," and "financial" areas.

\section{Limitations and Future Recommendations}

As this investigation is predominantly an explorative and pioneer examination of the utilization of telehealth services in addiction treatment in the state of Colorado, the data gathered are primarily descriptive in nature and therefore requires nonparametric analysis of data. Thus, in order to acquire a more nuanced understanding and develop a more comprehensive explanation 
regarding the relatively infrequent use of telehealth use in addiction treatment, future research is encouraged to investigate in more detail the identified barriers, as well as potential stigma and concerns of addiction professionals that appears to limit the utilization of telehealth services.

Our complete survey results are based on the replies of counselors that indicated that they do use telehealth interventions. To develop a more comprehensive understanding of existing barriers it is recommended that future research focus on creating a survey instrument to mine more in-depth information on the reasons counselors chose not to use telehealth in addiction treatment.

Several methodological concerns emerged during the course of this research. Since the survey link in this study was posted on the profile page of CAAP on LinkedIn, it is possible that some individuals with occupations other than addiction treatment clinicians may also have completed the survey. The total sample size for this study was also quite small, since only respondents answering that they are currently using telehealth in the treatment of addictions were routed to complete the remainder of the survey. In addition, attrition was also present in the current study, with some of the sample not completing the entire survey. Therefore, in order to enhance the validity and generalizability of the findings, it is suggested that future research should limit the study sample to clinical professionals in the addiction field only, and do what is necessary to increase the final sample size and response rate.

Lastly, to increase the understanding of and facilitate the willingness to utilize telehealth services in the substance disorder treatment field, further investigations are encouraged to examine the prevalence of and accessibility to telehealth trainings, as well as possible incentives to participate in such trainings. It is recommended that the development of telehealth trainings for addiction treatment be tailored to increasing its suitability for unique clinical health 
practitioner populations. For instance, it would be very helpful to explore potential ways of making telehealth technologies more "user-friendly" for clinicians from all age groups through added sophistication in technological design and increased specificity and relevance in the trainings offered. Identifying which specific technologies are best suited to meet the needs of unique addiction treatment providers, as well as clients, would cultivate interest in the provision of telehealth services. Determining strategies to attract practitioners to state-of-the-art training in telehealth technology is critical to maintaining currency in service delivery in the addiction treatment field. On a final note, gathering additional information on the comfort levels and preferences of clients would provide useful guidance in the development of future telehealth service methods. 
This research has been approved by the university's Institutional Review Board (IRB), Human Subjects Protection Program, Office of Research Compliance, and has followed procedures in accordance with the ethical standards laid down in the 1964 Declaration of Helsinki and its later amendments.

The voluntary nature of this study was stated clearly in the text and all persons gave their informed consent prior to their inclusion in the study.

\section{Conflict of interest}

None of the authors have conflicts of interest and authors have full control of all primary data and agree to allow the journal to review data if requested. 


\section{References}

American Psychological Association. (1997). APA statement on services by telephone, teleconferencing, and internet: A statement by the Ethics Committee of the American Psychological Association. Washington, DC: American Psychological Association.

Baca, C. T., Alverson, D. C., Knapp-Manuel, J., \& Blackwell, G. L. (2007). Telecounseling in rural areas for alcohol problems. Alcoholism Treatment Quarterly, 25(4), 31-45. doi: 10.1300/J020v25n04_03

Batastini, A. B., King, C. M., Morgan, R. D., \& McDaniel, B. (2016). Telepsychological services with criminal justice and substance abuse clients: A systematic review and meta-analysis. Psychological Services, 13(1), 20-30.

Benavides-Vaello, S., Strode, A., \& Sheeran, B. C. (2013). Using technology in the delivery of mental health and substance abuse treatment in rural communities: a review. Journal of Behavioral Health Services \& Research, 40:1.

Canale, N., Vieno, A., Griffiths, M. D., Marino, C., Chieco, F., Disperati, F., Andriolo, S., \& Santinello, M. (2016). The efficacy of web-based gambling intervention program for high school students: a preliminary randomized study. Computers in Human Behavior, 55, 946-954.

Carlbring, P., \& Smit, F. (2008). Randomized trial of internet-delivered self-help with telephone support for pathological gamblers. Journal of Consulting and Clinical Psychology,76(6), 1090-1094. doi:10.1037/a0013603

Curie, C. G. (2005). From the administrator: High-tech options expand horizons. SAMHSA News, 13(1). 
Enos, G. (2015). Insurance mandates for telehealth should inspire addiction field. Alcoholism \& Drug Abuse Weekly, 27(9). doi: 10.1002/adaw.30093

Evans, L., \& Delfabbro, P. H. (2005). Motivators for change and barriers to help-seeking in Australian Problem gamblers. Journal of Gambling Studies, 21(2), 133-155.

Gagnon, M. P., Duplantie, J., Fortin, J. P., \& Landry, R. (2006). Implementing telehealth to support medical practice in rural/remote regions: What are the conditions for success? Implementation Science, 1(18).

Health Resources \& Services Administration. (2012) Retrieved from: https://www.hrsa.gov/ruralhealth/telehealth/index.html

Knopf, A. (2016). 42 CFR Part 2 proposed rule would allow 'general' consent. Alcoholism \& Drug Abuse Weekly, 28(7), 3-5. doi:10.1002/adaw.30472

Leach, W. D. (2009). If you bill it they will come, a literature review on clinical outcomes, costeffectiveness, and reimbursement for telemedicine. California Telemedicine and eHealth Center.

Linkous, J. (2012). Challenges in telehealth. The role of telehealth in an evolving health care environment. Retrieved from https://www.nap.edu/read/13466

Marsch, L. A., Guarino, H., Acosta, M., Aponte-Melendez, Y., Cleland, C., Grabinski, M., Brady, R., \& Edwards, J. (2013). Web-based behavioral treatment for substance use disorders as a partial replacement of standard methadone maintenance treatment. Journal of Substance Abuse Treatment, 46(1), 43-51.

McCarty, D., Rieckmann, T., Baker, R. L., \& McConnell, K. J., (2017) The perceived impact of 42 CFR Part 2 on coordination and integration of care: A qualitative analysis. Psychiatric Services, 68(3), 245-249. 
Mehrotra, A., Huskamp, H. A., Souza, J., Uscher-Pines, L., Rose, S., Landon, B. E., . . Busch, A. B. (2017). Rapid Growth In Mental Health Telemedicine Use Among Rural Medicare Beneficiaries, Wide Variation Across States. Health Affairs,36(5), 909-917. doi:10.1377/hlthaff.2016.1461

Myrseth, H., Brunborg, S. G., Eidem, M., \& Pallesen, S. (2013). Description and pre-post evaluation of a telephone and Internet based treatment programme for pathological gambling in Norway: a pilot study. International Gambling Studies, 13(2), 205-220.

National Association of State Alcohol and Drug Abuse Directors, Inc. (NASADAD), (2009). Telehealth in state substance use disorder (SUD) services report. Washington DC: Jaclyn Sappah \& Rick Harwood.

Ohinmaa, A., Chatterley, P., Nguyen, T., \& Jacobs, P. (2010). Telehealth in substance abuse and addiction: Review of the literature on smoking, alcohol, drug abuse and gambling. Institute of Health Economics. Alberta, Canada.

Schaper, E., Padwa, H., Urada, D., \& Shoptaw, S. (2016). Substance use disorder patient privacy and comprehensive care in integrated health care settings. Psychological Services, 13(1), 105-109.

Smalley, K. B., Yancey, K. T., Warren, J. C., Naufel, K., Ryan, R., \& Pugh, J. L. (2010). Rural mental health and psychological treatment: a review for practitioners. Journal of Clinical Psychology, 66(5), 479-489. doi: 10.1002/jclp.20688

Qualtrics research core - Sophisticated research made simple. (2017). Retrieved from https://www.qualtrics.com/research-core/ 


\section{Appendix A \\ Telehealth Services Survey - For Individuals}

Survey Introduction. You might have noticed the expanding role that technology is playing in behavioral health. In order to construct a state-wide plan moving forward, it is important to define the current status of Telehealth utilization in Colorado. To this end, we are attempting to identify the current technology usage patterns in the field of addictions treatment in Colorado.

We greatly appreciate your input and support by completing this short survey. Study results will be provided to you upon completion of this research project.

\section{Section One: Background Information}

1. What is your job title or primary position?

O Administrator

Clinical Supervisor

Clinician

O Researcher

O IT

Others - Please Specify

2. Please identify your gender.

O Male

Female

Other - Please Specify

3. Please identify your age.

$O<30$ years

- 31-41 years

- 42-50 years

O 51-60 years

$0>60$ years 
4. Please select the ethnic group you identify the most with:

White

African American

O American Indian/Alaska Native

O Asian

Native Hawaiian and Native Pacific Islander

O Hispanic/Latino(a)

Other - Please Specify

Prefer Not to Identify

5. What type of organization do you work for? (Please check all that apply)

Addiction treatment program (inpatient, outpatient, residential)

$\square$ Hospital/Hospital affiliated Clinic

$\square$ School/University based Clinic

$\square$ Community Mental Health Center

$\square$ Tribal Health Clinic/Center

$\square$ Community Health Center

$\square$ Correctional Facility

$\square$ Military/VA

$\square$ Private Practice

$\square$ Other (e.g. Advocacy organization, Faith-based organization, Recovery Support Program, etc.) - Please Specify

6. What services do you offer?

$\square$ Substance abuse treatment

$\square$ Mental health treatment

$\square$ Recovery support services

$\square$ Other - Please Specify

7. Please provide the name of your agency.

8. Please provide the address of your agency, including zip code.

9. Where are your services provided?

Urban

O Rural 
10. Please rate the following.

\begin{tabular}{|c|c|c|c|c|c|}
\hline $\begin{array}{c}\text { What is your } \\
\text { comfort level } \\
\text { with } \\
\text { technology } \\
\text { use in your } \\
\text { professional } \\
\text { setting } \\
\text { (computer, } \\
\text { smart phone, } \\
\text { tablet, other } \\
\text { mobile } \\
\text { device)? }\end{array}$ & $O$ & $\mathrm{O}$ & $\mathrm{O}$ & $O$ & $O$ \\
\hline
\end{tabular}

11. How many years of experience do you have working in substance abuse treatment/recovery?

O Less than a year

O $1-5$

O $5-10$

O $10-15$

O $15-20$

O $20+$

12. I am currently using Telehealth technologies (e.g. computer, smart phone, tablet, other mobile device) to deliver services to my clients:

O Yes

O No 


\section{Section Two: Current Use of Telehealth Services}

* Please note that the use of telephone services should be referenced as Telehealth services only when counseling sessions were provided.

1. What forms of Telehealth technologies (treatment or communication) do you use? (Please select all that apply)

$\square$ Video Conferencing

$\square$ Instant Messaging

$\square$ Email

$\square$ Text Messaging

$\square$ Phone

$\square$ Mobile Apps

$\square$ Web-based interventions (i.e. learning modules, quizzes, activities, etc.)

$\square$ Other - Please Specify

2. How many years of experience do you have working with Telehealth?

Less than a year

○ $1-5$

O $5-10$

O $10-15$

O $15-20$

○ $20+$

3. How many years of experience do you have working with Telehealth in addiction treatment services?

Less than a year

O 1-5

O $5-10$

O 10-15

O 15-20

○ $20+$ 
4. How many years have Telehealth technologies been used at your workplace?

Less than a year

O $1-5$

○ $5-10$

O $10-15$

O $15-20$

○ $20+$

5. Which form of Telehealth technologies do you use most frequently to provide addiction services?

Synchronous (real time - e.g. video conferencing)

Asynchronous (store and forward - e.g. emailing)

6. Which of the following methods do you use most frequently? (Please select all that may apply)

$\square$ Video Conferencing

$\square$ Instant Messaging

$\square$ Email

$\square$ Text Messaging

$\square$ Phone

$\square$ Mobile Apps

$\square$ Web-based interventions (i.e. learning modules, quizzes, activities, etc.)

$\square$ Other - Please Specify

7. Which of the following methods do you use the least frequently? (Please select all that may apply)

$\square$ Video Conferencing

$\square$ Instant Messaging

$\square$ Email

$\square$ Text Messaging

$\square$ Phone

$\square$ Mobile Apps

$\square$ Web-based interventions (i.e. learning modules, quizzes, activities, etc.)

$\square$ Other - Please Specify 
8. Which of the following activities do you currently utilize with Telehealth technologies?

(Please select all that apply)

$\square$ Screening

$\square$ Assessment

$\square$ Counseling

$\square$ Continuing care

$\square$ Client education

$\square$ Recovery support

$\square$ Clinician/staff training

$\square$ Clinical supervision

$\square$ Case management

$\square$ Medical management

$\square$ Other - Please Specify

9. How are you reimbursed for providing services utilizing Telehealth technologies? (Please select all that apply)

$\square$ Do not receive reimbursement for Telehealth technologies

$\square$ Medicare

$\square$ Medicaid

$\square$ Private insurance

$\square$ Private pay

$\square$ Sliding scale

$\square$ Other - Please Specify

\section{Section Three: Other Information About Telehealth Services}

* Please note that the use of telephone services should be referenced as Telehealth services only when counseling sessions were provided. 
1. What topics were you trained on for delivering services using Telehealth technologies? (Please select all that apply)

$\square$ How to use equipment/technology

$\square$ Confidentiality issues (HIPPA; 42 CFR Part 2)

$\square$ Licensing and credentialing

$\square$ State-specific regulations/requirements

$\square$ Reimbursement documentation, storage, and retrieval of records

$\square$ Obtaining consent

$\square$ Client management

$\square$ Ethics and safe practices

$\square$ Other - Please Specify

2. How is monitoring and supervision handled? (Please select all that apply)

$\square$ Email

$\square$ Face-to-face

$\square$ Phone

$\square$ Other - Please Specify

3. What type of encryption are you using to protect client data? (Please select all that apply)

$\square$ None

$\square$ TLS

Hushmail

$\square$ Encrypting your computer

$\square$ Encrypting your phone

$\square$ Dual authentication

$\square$ Other - Please Specify 
4. What is your biggest concern with Telehealth services? (You may select more than one)

- Cost

$\square$ Training

$\square$ Billing/reimbursement

$\square$ Clinician/staff acceptance

$\square$ Client acceptance

$\square$ Using the technology and equipment

$\square$ Credentialing

$\square$ Confidentiality

$\square$ Other - Please Specify

5. The following are some potential barriers to using Telehealth technologies. Please rate each of the following by circling the perceived extent to which each serves as a barrier to using Telehealth technologies in delivering treatment and recovery services.

\begin{tabular}{|c|c|c|c|}
\hline Cost & $\mathrm{O}$ & O & $O$ \\
\hline Training & $\bigcirc$ & $\mathrm{O}$ & $\mathrm{O}$ \\
\hline Billing/reimbursement & $\bigcirc$ & $\mathrm{O}$ & $\mathrm{O}$ \\
\hline $\begin{array}{l}\text { Clinician/staff } \\
\text { acceptance }\end{array}$ & $\mathrm{O}$ & $\mathrm{O}$ & $\mathrm{O}$ \\
\hline Client acceptance & $\mathrm{O}$ & $\mathrm{O}$ & $\mathrm{O}$ \\
\hline $\begin{array}{l}\text { Using the technology } \\
\text { and equipment }\end{array}$ & $\mathrm{O}$ & $\mathrm{O}$ & $\mathrm{O}$ \\
\hline Credentialing & $\mathrm{O}$ & $\mathrm{O}$ & $\mathrm{O}$ \\
\hline HIPPA/42 CFR Part 2 & $\mathrm{O}$ & $\mathrm{O}$ & $\mathrm{O}$ \\
\hline
\end{tabular}

6. Please rate the following:

\begin{tabular}{|c|c|c|c|c|c|}
\hline $\begin{array}{l}\text { What is your } \\
\text { interest level in } \\
\text { using/continuin } \\
\text { g to use } \\
\text { telehealth? }\end{array}$ & $\bigcirc$ & $\bigcirc$ & $O$ & $\mathrm{O}$ & $\mathrm{O}$ \\
\hline
\end{tabular}


7. What other information would be helpful to enhance your understanding of Telehealth? Please specify.

8. What other information/assistance might you need to increase the likelihood for you to use Telehealth in the future? Please Specify. 